\section{STEREOGRAPHIC PHOTOGRAPHY ON THE COMPUTER}

\author{
Richard A. Blade \\ University of Colorado - Colorado Springs \\ Editor: Hal Thwaites
}

\begin{abstract}
The author provides a semi-technical review of the principles of stereographic photography usable without special equipment and discusses the current methods of viewing stereo photographs as computer images. Included is a discussion of recently developed commercial software called Wireframe Express that allows two or more photographic images of an object, taken at different but arbitrary positions, to be joined into a composite 3D model for stereographic viewing from any angle. Among other things, this recent technology allows the virtual reality simulation of historic buildings and rooms to be constructed from currently existing photographs. Illustrations of the points are made with images provided on the CD-ROM.
\end{abstract}

\section{History of Stereo Photography}

1839 is the usual date ascribed to the birth of photography by the Frenchman Daguerre, although successful experiments actually date back to 1827 [Mace, 1990]. The official birth of the stereograph ${ }^{1}$ is commonly given as 1851, with the Crystal Palace Exhibit in London, what might be called the first World's Fair. A more accurate invention date is probably 1849 , which is the publication date of an article describing the invention of Sir Charles Brewster.

Since that time tens of millions of stereographic photographs have been produced. The library of the University of California - Riverside has several million of the stereographs in its archives, and other libraries have large collections as well. Many famous photographs, including many from the Civil War and Powell's famous expedition through the Grand Canyon, were originally stereographic. For perhaps 70 years many homes and most libraries had stereo viewers which they used for entertainment much as people use TV today. Even today, a sizable fraction of the American children have Viewmaster stereo viewers.

Photographs of early stereo cameras are included on the CD-ROM \{\{stereo camera photos\}\}[Willsberger, 1977], [Williams, 1972]. Included as well are

\footnotetext{
${ }^{1}$ Also called stereotype, stereoview, stereoscopic view, and stereo card.
}

photographs of two stereo viewers \{\{stereo viewers\}\}, [Williams, 1972] and some early stereographs dating back to 1857 \{\{early stereographs\}\} [Nazarieff, 1985][Private collection].

\section{Making Stereo Photos}

As the reader no doubt knows, the basic idea behind stereo photography is to present slightly offset images to the left and right eyes. The difference in parallax then provides the mind with information about the relative distance from the viewer. Since the eyes are roughly 2.5 inches $(6.5 \mathrm{~cm})$ apart, ideally the two photos should be taken $6.5 \mathrm{~cm}$ apart. Stereo cameras take the two photos simultaneously, and special viewers are made for the stereo pairs thus produced. Moreover, special attachments are made for $35 \mathrm{~mm}$ SLR cameras that put a single stereo pair into an ordinary $35 \mathrm{~mm}$ frame.

However, neither a special camera nor an expensive viewer is necessary if you wish to make your own stereos pairs for viewing on a computer. First, you can take the two photos of a stationary scene sequentially, merely offsetting the camera after the first shot. It is critical that the optical axis of the second shot be parallel to that of the first shot. This applies even if the photos are being taken of a relatively close object.

The offset distance, called the base, is not critical. You can certainly make the base greater than $6.5 \mathrm{~cm}$, called hyper-stereo, and most people will interpret the distances on the basis of the subject matter. In the extreme, however, the view will appear as though you are looking at a miniature model of the scene. For example, if the two photos are taken with a base of $13 \mathrm{~cm}$, then in principle all the distances in the scene will appear collapsed by a factor of two. In some cases this may actually be desirable. For example, a colleague of mine took sequential photos of the grand canyon from an aircraft. The resulting stereo pairs give the impression of a miniature canyon, but it actually shows up the depth better than if a proper base had been used.

Typically the immersive impact of stereographic photos is best when one has a closeby object at a distance of about $2 \mathrm{~m}(6 \mathrm{ft})$ as well as a background at infinity. (Incidentally, if you have not looked at stereo photos for a while, you may discover that you will not experience much immersion for the first hour or two of viewing.) The socalled depth of field of a typical person is roughly $2 \mathrm{~m}$ to infinity. It is not natural for people to perceive any larger depth of field than that, and since accommodation (changing the focal length of the eye lens by muscle action) is not available in a stereo view, views of closer objects, at least from a standard base, are disconcerting. 


\section{Viewing Stereographs on the Computer}

In the past the greatest problem with stereo photography without special equipment was finding or making a special viewer that is compatible with the stereo pairs (or making the stereo pairs compatible with a standard viewer). Using computers for viewing, this problem is largely eliminated.

There are basically three methods of viewing stereo images from a computer. The simplest and easiest is to use red-blue colored glasses to view an anaglyph image that has been properly prepared. Anaglyph viewing glasses are included with the CD-ROM so that you can view the stereo images without additional equipment. More on that shortly.

Another relatively inexpensive solution is shutter glasses. These glasses alternately blank the two eyes in synchrony with alternately displayed left and right eye images. Shutter glasses operating at low frequencies (approximately $30-50 \mathrm{~Hz}$ ) tend to produce a flicker, though that may be minimized by turning down the intensity. At higher frequencies $(60-120 \mathrm{~Hz})$ there is no noticeable flicker even at higher intensities. Low cost shutter glasses are available from 3DTV for roughly $\$ 150$, and are available for IJVR readers at a discount. ${ }^{2}$ Higher quality (and frequency) shutter glasses are available for something like $\$ 500$ from manufacturers such as Stereographics Corp.

The third major category of stereographic viewers for the computer is the head mounted display (HMD). The typical HMD has significant advantages over the other two methods, though at a significantly greater price tag. The primary advantage is that the HMD typically has a much larger field of view. Immersion increases dramatically for larger viewing angles, and the combination of stereo and wide viewing angle provides a spectacular experience!

Because the latter two methods of viewing stereo pairs are dealt with by hardware and software that is specific to the manufacturer, I shall not deal with those techniques further. It is sufficient to say that both shutter glasses and HMDs use separate left and right stereo images. A few left-right image files are provided on the CD-ROM as .gif files for those readers who have the proper viewing equipment.

\section{The Process of Creating Anaglyphs}

Fortunately, any number of graphics software packages have the capability of making anaglyph images. Among them are the commercial packages Adobe Photoshop, Aldus Photostyler, and the shareware package PICLAB. I prefer the inexpensive software from

\footnotetext{
2 3DTV Corp. is a corporate sponsor of the IJVR and makes shutter glass kits available at a discount. See this in the Sponsors section of this issue.
}

Synthonics called 3 DMaker, ${ }^{3}$ which is specially designed to create the anaglyphs from left-right stereo images. All you have to do is open the left image on the left side of the screen, the right image on the right side of the screen, choose the point on each that will coincide in the final image, and click the mouse on "Make3D" to complete the process.

Choosing the point of coincidence on each image that will coincide in the final anaglyph takes some trial and error. The directions say that the point may be considered as a point on the computer screen as though you are looking through the screen as a window. While you can let a point on the border of the picture be at the screen distance, points within the scene are typically much further away. Nonetheless, making trial anaglyphs is a rapid process with Make $3 \mathrm{D}$, so the process is quite painless. I present several anaglyphs of the same image on the CD-ROM to show the difference in the point of coincidence. \{\{stereo images\}\} One can observe that even a 0.1 inch displacement of the coincidence point to the right in the left screen image makes a great difference, and double that is almost unviewable ("unfusable"). A displacement of even 0.1 inch in the opposite direction renders an unviewable image, as it ries to force the eyes to view an image behand the head.

It is usually impractical to make images appear to be in front of the computer screen unless they are small. If the border of the screen, which has to be perceived to be behind the object, cuts off any portion of that image, the result will be a visual incongruence.

\section{Color Considerations for Anaglyphs}

I think that it is remarkable that if a person sees red through one eye and blue through the other, the brain integrates these into white, just as if each eye were seeing both colors. There does arise a perceptual problem, however, when the colors on the original image are sufficiently saturated that only one eye sees a significant portion of an image. That image or portion thereof is then referred to as a "ghost". Large areas of saturated colors, particularly reds and greens, typically cause ghosts. \{\{stereo images with a ghost\}\} Blue is not as much of a problem because the human eye is only $1 / 3$ as sensitive to blue as to red, and only $1 / 6$ as sensitive to blue as to green. [Pesce and Rogues, 1994] The remedy for ghosts caused by large areas of staturated colors is to significantly lessen the contrast or even modify the colors if that can be done without ruining the realism. I include some \{\{stereo images\}\} with varying degrees of color saturation to illustrate the differences.

\footnotetext{
3 Synthonics is also a sponsor of the IJVR, and the IJVR provides its readers with a discounted price on this software. See the Sponsors section of this issue for details.
} 


\section{Borders}

The border of the stereo image is typically black, and in an attempt to make sense out of the image within, the borders are sometimes perceived as inconguent. That is, the brain creates colored ghosts on the borders. (See \{\{stereo image borders\}\}. Such ghosts are remedied by cutting down the borders of the image until the left and right images appear to have the same border, preferably at the perceived location of the computer screen.

\section{Stereographs from Nonstereo Photos}

As I mentioned in the abstract, there exists new software from Synthonics, called Wireframe Express, ${ }^{4}$ that can take two or more very different views of a scene or object and integrate them into a stereographic view from (almost) any direction. You click on the common points in the photographs and the software constructs polygons on a three-dimensional model that you or the software maps textures onto. Clearly areas that are not covered by at least two of the photos cannot be accurately mapped, so such regions must be left out of the final image. A series of $\{$ images $\}\}$ on the CD-ROM illustrates.

As in 3DMaker, the Wireframe Express software requires some trial and error to get, say, vertical walls to be vertical. After the three dimensional model has been generated, however, it is possible to generate views from any angle, including stereo views and even a stereo walkaround.

I include on the CD-ROM a number of \{\{stereo images\}\} that illustrate the use of the Wireframe Express software, as well as a .fli animation file \{\{animation\}\} for those equipped to view it.

\section{Conclusions}

Stereo photo buffs will enjoy the expanded capabilities of generating stereo images on the computer. Because of the ease of integrating and modifying computer images with special software, many of the difficulties encounted in the past with stereo photography will be eliminated.

\section{REFERENCES}

Adams, Ansel, The Camera (Book 1 of The New Ansel Adams Photography Series), New York Geographical Soc., 1980.

Mace, O.H, Collectors Guide to Early Photographs, Wallace-Homestead, 1990.

Pesce, Bruno, and Rogues, Silvain, DO-3D, 1994.

(This is a freeware tutorial on stereography. The

\footnotetext{
${ }^{4}$ As in the case of 3DMaker, because Synthonics is a sponsor of this joumal, readers may order the software through us at a discounted price. See the Sponsors section for details.
}

entire package, including drawings and images, is viewable from the CD-ROM: $\{$ (Do-3D $\}\})$

Nazarieff, S., Stereo Nudes, 1850-1930, Editions Filipacchi-SONODIP, Paris, 1985.

Williams, R., ed, Frontiers of Photography, Time-Life Books, 1972.

Willsberger, Hohann, The History of Photography, Doubleday, 1977.

In addition, I have included a significant number of stereographs from a private collection (owner wants to be anonymous), from a CD-ROM from $3 D T V$ Corp., and from a collection of stereographs provided me by Synthonics Corp.

\section{BIOGRAPHY}

RICHARD BLADE \{\{photo\}\} is a Professor of Physics at the University of Colorado - Colorado Springs. Other professional positions include Caltech, University of California - Berkeley, and United States International University. Professor Blade serves as science editor for IPI Press and Editor-in-Chief of the IJVR. He also serves as Focus Chair for IEEE Standards in Virtual Reality and Chair of the Working Group on the VR Glossary. His current research involves VR motion simulation.

Contact information:

Richard A. Blade

IPI Press

2608 N. Cascade Ave.

Colorado Springs, CO 80907

Pers Phone: 719-471-4476

FAX: 719-630-1427

Email: rblade@mail.uccs.edu 